\title{
Raman Spectroscopy for Homeland Security Applications
}

\author{
Gregory Mogilevsky, ${ }^{1}$ Laura Borland, ${ }^{1}$ Mark Brickhouse, ${ }^{1}$ and Augustus W. Fountain III ${ }^{2}$ \\ ${ }^{1}$ Booz Allen Hamilton Inc., 4692 Millennium Dr., Suite 200, Belcamp, MD 21017, USA \\ ${ }^{2}$ Research and Technology Directorate, Edgewood Chemical Biological Center, 5183 Blackhawk Road, \\ Aberdeen Proving Ground, MD 21010-5224, USA \\ Correspondence should be addressed to Augustus W. Fountain III, augustus.w.fountain.civ@mail.mil
}

Received 20 February 2012; Accepted 16 April 2012

Academic Editor: Kevin Spencer

Copyright ( $) 2012$ Gregory Mogilevsky et al. This is an open access article distributed under the Creative Commons Attribution License, which permits unrestricted use, distribution, and reproduction in any medium, provided the original work is properly cited.

\begin{abstract}
Raman spectroscopy is an analytical technique with vast applications in the homeland security and defense arenas. The Raman effect is defined by the inelastic interaction of the incident laser with the analyte molecule's vibrational modes, which can be exploited to detect and identify chemicals in various environments and for the detection of hazards in the field, at checkpoints, or in a forensic laboratory with no contact with the substance. A major source of error that overwhelms the Raman signal is fluorescence caused by the background and the sample matrix. Novel methods are being developed to enhance the Raman signal's sensitivity and to reduce the effects of fluorescence by altering how the hazard material interacts with its environment and the incident laser. Basic Raman techniques applicable to homeland security applications include conventional (off-resonance) Raman spectroscopy, surface-enhanced Raman spectroscopy (SERS), resonance Raman spectroscopy, and spatially or temporally offset Raman spectroscopy (SORS and TORS). Additional emerging Raman techniques, including remote Raman detection, Raman imaging, and Heterodyne imaging, are being developed to further enhance the Raman signal, mitigate fluorescence effects, and monitor hazards at a distance for use in homeland security and defense applications.
\end{abstract}

\section{Introduction}

There are a number of factors driving research, development, and acquisition of new technologies for detecting chemical, biological, radiological, nuclear, and explosive (CBRNE) threat materials. The addition of new and emerging threats has increased in recent years and, with these new agents, the number and type of physical properties to be detected have also increased. Historically, the Department of Defense prepared to defend against the mass use of war gases, such as chlorine or phosgene, or less volatile nerve and blister agents (Sarin, sulfur mustard, etc.), which could still present a substantial vapor hazard under certain environmental conditions. More recently, low volatility nerve agents, such as VX, or aerosol-based biological agents, such as Bacillus anthracis (anthrax), became the prevalent threats and increased the need for robust aerosol and point detection capabilities to detect these agents. As the potential for Cold War-style engagements of massed armies with large quantities of weaponized chemical and biological agents receded, the need has increased to defend against and deter small-scale attacks of increasingly toxic emerging chemical and biological (CB) agents from rogue states, nonstate parties, or terrorist groups. This change in the $\mathrm{CB}$ threat has been accompanied by the expanding use of improvised explosive devices (IEDs) by adversaries and the increasing need to detect explosives left-of-boom, to reduce IED casualty rates. These changes in prevailing threats present a substantial challenge for chemical agent and explosives detection. Detection of a high concentration of a small number of vaporous agents has given way to the need to detect a large number of less volatile agents, often on surfaces and at lower concentrations. Additionally, the desire to Detect to Protect rather than Detect to Treat increases the need for rapid, real-time analysis of threats in the field, to minimize casualties and reduction in operational tempo (OPTEMPO). Finally, the increasing number of sensors in the field, combined with the general logistical burden to soldiers and emergency responders, is 
driving the need for the convergence of sensor capabilities and for detectors that can identify a wide range of threats agents.

This paper addresses the applicability of a particular laser-based optical technique, Raman spectroscopy, to emerging CBRNE detection needs. Raman spectroscopy is based on the inelastic scattering of monochromatic near infrared (NIR), visible, or near ultraviolet (UV) light, which reveals low-frequency vibrational and/or rotational modes in target analytes [1]. Raman spectroscopy is an inherently insensitive technique (Raman light scattering is typically weak) that has benefited enormously from recent developments in laser light sources. In addition, fluorescence off the sample or from the sample environment obscures the signal. More sensitive optical detectors and improved data processing algorithms are being developed to mitigate these issues [2-5]. As such, Raman spectroscopy is transitioning from purely lab-based applications to more challenging fieldbased applications, such as detection of emerging threat agents in realistic environmental settings. This paper is not intended to be an in-depth chronicle of all available Raman spectroscopy methods and their histories. Rather, it highlights recent experiments and methods that would be relevant for defense and homeland security applications.

\section{Challenges in Detection and Identification of Homeland Security and Defense Threats Using Raman Spectroscopy}

Raman spectroscopy is a well-established technique for identifying potentially hazardous or illegal substances in the homeland security and defense arenas $[6,7]$. Raman techniques are nondestructive and can be implemented in a laboratory, using state-of-the art spectrometers, or in the field, using portable devices [8]. Homeland security applications typically require that samples be identified positively, and nondestructively, so that the composition of the original sample interrogated in further laboratory tests and remain intact and available as potential evidence. Raman spectroscopy avoids physical contact with the sample, preserving sample integrity.

The main physical difference between Raman spectroscopy and other optical techniques, such as UV-Visible absorption or fluorescence spectroscopy, is that the Raman signal arises from scattered light, rather than from absorbed and/or emitted photons [9]. As a consequence, the Raman scattering effect is independent of the incident laser wavelength. The Raman modes are unique to specific chemical groups, corresponding to molecular vibrations of a molecule. Because of this, Raman spectroscopy is often referred to as "molecular fingerprinting" $[8,10,11]$. In some cases, other spectroscopic techniques produce false positive results. For example, fluorescence detection yields false positive results because fluorophore groups produce emission bands that can be misinterpreted [12]. In contrast, Raman spectra have sharp lines, which correspond to unique and specific chemical species and do not require extensive specimen preparation. Raman spectroscopy does not require fluorophore staining, as required for fluorescent spectroscopy of nonfluorescent chemical and biological samples. Furthermore, Raman can be used to identify substances in all states of matter; solid, liquid, and gas; as well as directly in ambient air or within transparent or opaque containers $[1,8,13-17]$.

The following sections outline strengths and weaknesses of individual Raman techniques. Emerging technologies, which strive to provide safe, accurate, and precise identification of materials with adequate signal-to-noise ratios in ambient or laboratory conditions, are also presented. While no one Raman technique is perfect in all possible applications, various forms of the techniques may be used together to analyze complex materials in complex matrices. Each discussed technique is unique in the way it further enhances the Raman spectroscopy signal by exploiting various physical properties of the sample interaction with the environment and laser excitation. Techniques that do not alter the physics of Raman detection and only enhance the signal by unique optics are discussed in the application section.

\section{Basic Raman Spectroscopy Techniques}

3.1. Conventional (Off-Resonance) Raman Spectroscopy. Conventional Raman spectroscopy is implemented in many defense and homeland security applications to identify potential threats and hazards. Here, we define conventional Raman spectroscopy as the collection of the Raman signal in backscattered geometry with no further enhancements or modification to the placement of the sample, incident laser, or the scattered Raman signal. In its most traditional form, conventional Raman was found to be excellent for field and laboratory forensic analysis. For example, Raman spectroscopy has been used for fingerprint analysis, to identify polymerized cyanoacrylate in fixed fingerprints and to detect foreign bodies trapped in the fingerprint, such as drugs or explosives $[18,19]$. This important forensic tool delivered qualitative results, which identified substances in an undisruptive manner. Additionally, conventional Raman signals can be transmitted through clear glass containers and have been used to monitor their contents without perturbing the sample [8].

While infrared (IR; $\lambda \sim 3-5 \mu \mathrm{m}$ ) spectroscopy techniques are highly sensitive to water, which produces a strong IR signal, Raman spectroscopy is insensitive to water, making the technique suitable for detection of biothreats [20] or hazardous and illegal substances dispersed in aqueous media [8]. Raman spectroscopy does not suffer from the issues of IR analysis of aqueous samples, which requires deconvolution of data from background water and special care, such as dry nitrogen purging of cellular samples prior to analysis [21]. In fact, Raman spectroscopy was used to detect part per million (ppm) levels of Raman-active salts in simulated sea water with no sample preparation or manipulation [22].

Conventional Raman spectroscopy is not without certain shortcomings. The most persistent interferences with Raman data are the high intensity background fluorescence signals, which can exist in collected Raman spectra. The fluorescence signal comes from photon emission due to inadvertent laser 
excitation. This is caused by the incident laser inducing charge transfer in the analyte, the sample matrix, or from impurities within the sample or in the environment $[1,23$, 24]. To experimentally mitigate these fluorescence effects, a laser line is chosen in the NIR $(700 \mathrm{~nm} \leq \lambda \leq 2000 \mathrm{~nm})$ or deep UV (DUV; $\lambda \leq 250 \mathrm{~nm}$ ) ranges, both of which reduce the negative effects of fluorescence. Even though the Raman intensity depends on the incident wavelength $\left(\sim \lambda^{-4}\right)$, the lack of fluorescence at longer wavelengths allows for a weak scattered signal to be measured [9]. While NIR Raman is primarily used in the medical field $[25,26]$, DUV Raman is widely applied in homeland security applications and will be discussed in the following sections. Finally, most of the signal in conventional, backscatter geometry Raman spectroscopy arises from the surface of the sample; therefore monitoring the constituency of the sample using a conventional Raman spectrometry is not possible [11]. Many of these shortcomings are handled by further enhancing the sample, incident laser beam, or the geometry at which Raman signal is collected.

3.2. Surface-Enhanced Raman Spectroscopy (SERS). The surface-enhanced Raman spectroscopy (SERS) enhancement effect occurs when Raman spectroscopy is performed on an analyte that is absorbed on the roughened surface of certain metal substrates $[27,28]$. Although the exact nature of SERS enhancement is still under debate, it is believed that most of the enhancement (up to $10^{14}$ ) comes from electric field enhancement occurring at the surface of the metallic substrate [15]. The electric field enhancement yields a stronger Raman interaction, originating from laser-induced surface plasmons on the patterned metal films [27, 29]. Because analytes are confined to the surface, the enhanced electromagnetic fields near the rough, nanoscale features contribute to the enhanced Raman signal $[6,16]$. Those areas on the substrates where the enhancement occurs are referred to as hot spots. In addition to achieving signal enhancement, nanoengineered SERS substrates provide a fluorescence quenching pathway to promote Raman signal collection, while minimizing the background [16].

More than other Raman spectroscopy techniques, the success of the SERS profoundly relies on advances in nanotechnology. The size and shape of the substrate features affect the enhancement of the SERS signal [30-32]. For optimum Raman signal enhancement, the feature sizes must be smaller than the wavelength of the incident radiation $[33,34]$. During the initial decades of research into the SERS effect, there was theoretical development and demonstration of Raman signal enhancement. First generation SERS technology did not contain materials engineered on the nanoscale [6]. Many initial studies on electrodes used adventitiously created nanostructures. These included randomly distributed features, made by roughening the metal surface or by randomly distributing metal colloids. More recent studies, patterned SERS substrates were created using electron-beam lithography $[35,36]$, focused-ion beam $[37,38]$, capillary force adhesion [39-42], and templating block copolymers. Many of the approaches documented in the references produced enhancement factors in the range of $10^{8}-10^{12}$ [43]. Unfortunately, these approaches failed to produce consistent and reproducible signal enhancements. They also deliver functional areas of less than $0.1-5000 \mu \mathrm{m}^{2}$, areas too small for practical devices and mass production [44].

Recent advances in fabricating techniques have led to the development of substrates with regular and reproducible nanoscaled features and sensitivity towards difficult to detect materials, such as explosives [41, 42, 44-47]. For example, TNT and DNT molecules were detected down to 0.1$0.05 \mathrm{ppt}$ levels using porous gold arrays as SERS substrates [48]. These arrays were made using photonic crystal fibers, which acted as waveguides for light and yielded high Raman signal enhancement. They were also made in a reproducible fashion, formed from long, identical fibers [49]. To further induce reproducibility of the SERS measurement, several studies implemented a commercially available substrate, Klarite, from D3 Technologies Ltd., to detect chemical and biological agents [50-54]. Unfortunately, sensitivity was sacrificed because the mass-produced features of the substrates were not ideal for all applications and contained a standard deviation error of up to $15 \%[55,56]$.

3.3. Resonance Raman. In conventional Raman spectroscopy, the incident laser photon stimulates the molecule into a virtual excited state and measures the resulting scattered light. If the incident laser's energy is close to an electronic transition within a molecule, such as a chromophore, the Raman signal is amplified by many orders of magnitude [9]. This effect is known as resonant enhanced Raman. Unfortunately, since the excitation occurs near an electronic absorption level and the Raman signal is inherently weak, additional fluorescent emission can overwhelm the Raman signal.

An optimal union of conventional off-resonance Raman spectroscopy and resonance Raman occurs when single band UV-lasers are used to probe for explosives [57-60]. Resonance was achieved in TNT and other explosive molecules using a single line UV or DUV laser. The absorbance peak in TNT is centered at $250 \mathrm{~nm}[60,61]$; therefore resonance enhancement can occur with a UV or DUV laser that operates at that wavelength. Contributions of any luminescence signal could be ignored during UV excitation because, in many cases, the fluorescence signal was outside of the Raman spectral range [59]. With newly developed background subtraction schemes, trace amounts $\left(55 \mu \mathrm{g} / \mathrm{cm}^{2}\right)$ of material can be detected, making the resonance Raman ideal for detection of low volatility and low abundance materials, such as explosives [58]. The UV-Resonance Raman (UVRR) combination approach can also be used to detect many different types of complex species by exploiting UVRR's ability to selectively probe specific modes of lipids and peptides $[62,63]$.

One approach that seeks to take advantage of the increased selectivity of resonance Raman spectroscopy is to use fully tunable, multiline lasers to probe a sample. To implement the technique in the field, these multiline lasers must be portable and rugged enough to be used in a handheld operation. Recently, photonic crystal fibers were used 
to generate portable, multiline pump lasers that could be implemented in portable resonance Raman spectrometers [64].

3.4. Coherent Anti-Stokes Raman Spectroscopy. To enhance the inherently weak Raman signal, a four-wave technique was developed, called coherent anti-Stokes Raman spectroscopy (CARS) [65]. The analyte molecule is put into a coherent oscillation by two pulsed incident laser beams, labeled the Stokes beam and the pump field beam, with frequencies $\omega_{s}$ and $\omega_{p}$, respectively. The beams are spatially focused tightly on the same spot in the sample. A third beam, the probe beam with frequency $\omega_{p r}$, is scattered off the vibrating molecule, inducing an anti-Stokes fourth beam (frequency $\left.\omega_{a s}=\omega_{p}-\omega_{s}+\omega_{p r}\right)$, which is measured. The enhancement of the Raman signal occurs when the $\omega_{p}-\omega_{s}$ frequency is the same as a molecular vibration level $[66,67]$. In typical experimental conditions, the pump laser is set to $\omega_{p}=\omega_{p r}$, making the signal frequency $\omega_{a s}=2 \omega_{p}-\omega_{s}$. The Stoke beam frequency is swept across the frequency range until the CARS signal is maximized [67]. Fluorescence problems that plague other Raman techniques are avoided naturally because the CARS signal is blue-shifted relative to the incident beam, whereas one-photon fluorescence background is red shifted [17].

Although originally described in 1970s, most of the technological advances in CARS have occurred since late 1990s and early 2000s [17]. Original CARS designs were hindered by numerous factors, such as weak induced polarizability and a high background from the nonresonant Raman signal. These challenges were addressed by developments of ultrafast (femtosecond) lasers and methods to suppress the background signals [68]. Many different variations on this technique currently exist in the literature.

CARS is a superior method to conventional Raman spectroscopy for trace explosive vapor detection. For a reasonable laser intensity ( $2 \mathrm{MW} 532 \mathrm{~nm}$ with $0.2 \mathrm{MW}$ dye laser power), signal efficiency is on the order of $10^{11}$ higher than conventional Raman for the measurement of gases. CARS efficiency drops as laser power increases and with increasing saturation of analyte [67]. Several groups implementing a single short-pulsed laser, instead of the traditional multiline method, detected trace amounts of various materials such as explosives, even at remote distances [69-73]. Using a single laser line eliminated the need to synchronize multiple laser lines; however, a large nonresonant signal background was introduced. Interestingly, the nonresonant background signal occurred with a well-defined phase offset from the resonant signal, allowing the resonant signal to be measured together with the nonresonant signal in the heterodyne [71]. The heterodyne signal was detected and the fluctuation in the nonresonant background was deconvoluted to produce the remnant resonant signal. This method was applied to standoff Raman applications for defense, specifically for the detection of trace amounts of solids (including RDX explosives) at a distance of 10 meters [69].

3.5. Temporally and Spatially Offset Raman Spectroscopy. A major drawback of scattered laser spectroscopic techniques is that the measurement is generally restricted to the light scattered off the sample surface. For example, typical Raman spectroscopy apparatuses are positioned to collect data in backscatter mode, where the excitation laser shines onto a sample and the scattered Raman signal is collected through the same aperture. This geometry is only able to collect data from the surface of the sample and does not give any information about the material's bulk or interior details. In many practical cases, such as the detection of materials encased in opaque containers, for example, narcotics, the examined surface is not indicative of the material of interest and penetration depths of millimeters or even centimeters are required. Moreover, when searching for counterfeit medicine, many capsule coatings produce an intense fluorescence spectrum, interfering and overshadowing any Raman signal $[74,75]$. To overcome these difficulties, it is possible to take advantage of the fact that some incident laser photons are able to penetrate the surface, induce the Raman effect in the interior of the material, and emerge either in the front or back side of the sample. These emerging photons contain Raman-shift information from the interior of the sample. This technique provides a powerful mechanism to profile interiors and build depth profiles of materials on millimeter to centimeter-length scales.

Additionally, light emerging from deeper areas of the sample will take a longer time to emerge, since it had to travel a longer distance than light exiting from more shallow regions. The light will also emerge from the sample further away from the incident laser spot since the photons have spread out further in the sample before emerging from the surface (Figure 1). This Raman phenomenon occurs on a timescale of 0.1 picosecond and can be considered instantaneous in these applications, especially relative to how long it takes for light to exit the interior of the sample [76]. By measuring the temporal and spatial characteristics of the emerging light, a depth profile map of the chemical composition for the sample is built. As shown in Figure 2, spectra taken at larger $r$ values correspond to deeper levels below the surface. Also, the photons at larger $r$ values will emerge later than those with smaller $r$ values, since they have to traverse a longer distance inside the sample. The technique that selects emerging photons based on time is called temporal offset Raman spectroscopy (TORS). The technique that measures the spatial distance of emerging photons from the source is called spatially offset Raman spectroscopy (SORS). They both measure light with the longest path diffused through sample [75]. These photons are capable of penetrating several centimeters, depending on the medium. From the time of their initial discovery, researchers demonstrated the offset techniques in homeland security-related applications, such as detecting biomaterials, pharmaceuticals, and other hazardous or potentially dangerous materials enclosed in various containers $[11,13,14,74,77-81]$.

\section{Applications of Raman Spectroscopies for Homeland Security and Defense}

The following section outlines emerging Raman spectroscopy techniques for Homeland Security and Defense 


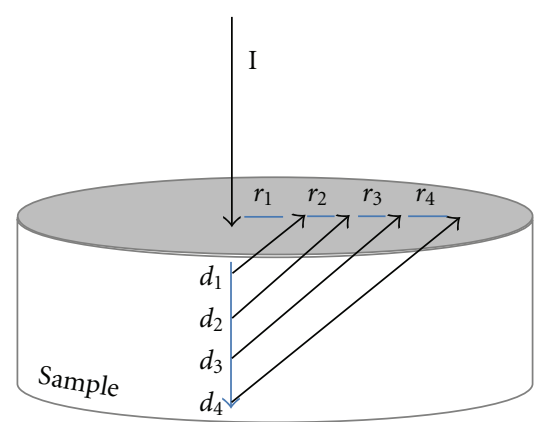

Figure 1: Offset Raman spectroscopy schematic. $r_{i}$ (distance from incident beam $I$ ) correspond to $d_{i}$ (depth). One can build depth profile of sample. Larger $r$ distances correspond to deeper levels.

applications that build upon the basic techniques outlined in the previous section. Advancements in laser and detector technologies allow for precise Raman measurements to be made in laboratory and field environments. With the introduction of the Volume Bragg Grating (VBG), portable lasers now deliver a stable, monochromatic excitation signal without mode hopping $[8,82]$. Also, sensitive thermoelectrically cooled charge-coupled device (CCD) detectors collect scattered Raman signals, even in ambient conditions [10, 83]. Further achievements in laser and detector technologies allow for excellent matching and distinguishing of chemicals using portable instruments. In portable systems, a quality match index of 0.01 is achievable [8].

4.1. Portable Raman Spectrometry. Several recent reviews $[84,85]$ and technology assessments [86] of portable optical spectrometers addressed the basic configuration and capabilities of portable Raman spectrometers commercialized by a number of firms in the last ten years. A typical portable Raman instrument consists of a compact NIR solidstate laser source, an optics block with bandpass filters, a dichroic mirror and lens, a spectrometer to disperse the Raman spectrum, a multichannel detector, typically a silicon charge-coupled device (CCD) or less sensitive nonsilicon NIR detector arrays (Ge, InGaAs), and a computer. Diode lasers are the most common source due to cost, weight, and, if the portable Raman spectrometer is operating at longer wavelength excitation, fluorescence mitigation. Fieldportable Raman instruments of a dispersive type have been developed and marketed by Ahura Scientific, B\&W Tek, Algitron, DeltaNu, Enwave Optronics, Identicheck, InPhotonics, Ocean Optics, PerkinElmer, Raman Systems, Renishaw, Senspex, and Smiths Detection.

Portable Raman spectrometers are typically used to identify unknown bulk samples, also known as the white powder problem. To illustrate the application of a portable Raman spectrometer in a setting outside of the laboratory, a research group set up experiments at a major airport and were able to identify a "street sample" of narcotics containing drugs in a matrix of cutting agents [10]. The sharp shapes of the Raman lines corresponded to unique chemical species. Since the Raman lines were unique and well

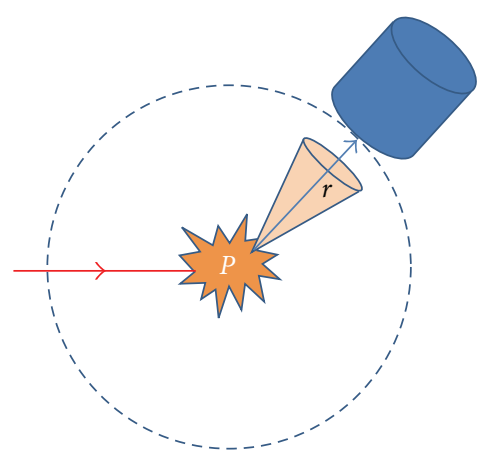

Figure 2: Detector spaced distance, $r$, away from analyte will collect a Raman spectrum whose intensity is reduced by a factor of $1 / r^{2}$.

defined, multiplex detection of specific chemical species in background matrices was achievable $[6,16]$. This is especially important for analysis of samples in the field, since the collected data often contains background signal from the environment.

The miniaturization of Raman instrumentation for portable devices reduces the ability to acquire the same quality results as are achievable in a laboratory instrument. For example, achieving high spectral resolution is a challenge. Some systems have registered resolution of $8-12 \mathrm{~cm}^{-1}$, which may be insufficient for samples whose linewidth is on the order of $5-10 \mathrm{~cm}^{-1}$. Careful calibration has to be performed to overcome drift of the $x$-axis (frequency) that comes from for factors such as temperature and humidity [8]. Also, it is challenging to distinguish components of a mixture using a portable system. However these technical challenges can be overcome and the benefit is the ability to detect hazards such as explosives in the field [85].

4.2. Remote Raman Spectroscopy. There are numerous missions in the field which require detection of unknown, potentially hazardous materials at a distance. In case of potential explosives, Raman spectroscopy must accurately and precisely identify the hazard at distances of at least 10-100 m [87]. Fortunately, Raman spectroscopy can be implemented on samples from distances of millimeters to greater than 50 meters [88]. The primary complications that arise out of remote Raman detection include reduced intensity of returning light, with light intensity decreasing as the distance squared, and loss of signal due to absorbed and scattered light in the atmosphere [87]. This problem can be described in terms of detected analytes having a limited photon budget, which is affected by the environment and the properties of the equipment. The photon budget is defined in the following equation, which relates the total number of Raman scattered photons collected by a detector (whether up close or at a distance) to the Raman cross section and environmental effects:

$$
N_{R}=\int_{0}^{D} N_{0} \eta_{t} \frac{d \sigma}{d \Omega} n_{\text {chem }} e^{-2 k R_{s}} \Omega(r) 10^{-\left(\alpha_{i}+\alpha_{R}\right) r} d r
$$

$N_{R}$ is defined as the total number of Raman-scattered photons collected by a detector for a given mode in realistic 
conditions as defined by $R_{s}$ (atmospheric path between the detector and the examined surface), $k$ (atmospheric extinction coefficient, independent of the Raman wavelengths), and the $\Omega(r)$ term (solid angle between the detector and the sample). $N_{0}$ is the number of incident laser photons. The remaining terms are independent of the environment and are related only to the analyte. The $n_{\text {chem }}$ term is the molecular density of the specimen. The $d \sigma / d \Omega$ term is the differential Raman scattering cross section, which is defined as the fraction of the total incident laser photons, scattered, per molecule irradiated into a solid angle. $D$ is the thickness of the sample, or the penetration depth of the laser. The $\alpha_{i}$ and $\alpha_{R}$ terms are the absorption coefficients of the specimen at the incident and Raman irradiation frequencies, respectfully. This is the total number of photons that an analyte can produce for given irradiation.

In addition to the limited photon budget that puts an inherent cap on how many photons are capable of detection, the distance between the detector and the analyte presents another challenge to overcome. The illustration in Figure 2 depicts an analyte remotely irradiated with an excitation laser. The induced Raman signal power $(P)$ from the sample will be distributed in space among a spherical area, $A(r)$, where a detector-positioned distance, $r$, away from the analyte will receive the signal with intensity, $I(r)$, as defined by.

$$
I(r)=\frac{P}{A(r)}=\frac{P}{4 \pi r^{2}} .
$$

The collected signal at distance is reduced from the initial intensity $(P)$ as a factor of $1 / r^{2}$.

The North Atlantic Treaty Organization (NATO) defines three detection distances from potential hazards. They are point, proximal, and standoff detection. Point detection refers to detection of hazards less than $10 \mathrm{~cm}$ away. Standoff distance, as defined by the NATO guidelines, refers to hazards located further than 200 meters away [89]. The intermediate distance, $10 \mathrm{~cm}-200 \mathrm{~m}$, is defined as proximal distance $[89,90]$. In the literature, Raman spectroscopy techniques at any distance away from the sample, typically over several centimeters, are referred to as "standoff" [72, 73, $88,91,92]$. True "standoff" detection, as defined by NATO, is nearly impossible to achieve using conventional Raman spectroscopy because of the weakness of the generated Raman signal. However, in most practical applications, detection at the proximal distance is desired, since awareness and detection of hazards will most likely be required at these distances. In this paper, we will refer to remote Raman detection as "proximal" Raman spectroscopy to adhere to NATO guidelines.

Typical proximal Raman spectroscopy equipment shares some basic components. A portable laser source is used to irradiate the offset sample with a laser beam. The generated Raman signal is captured and magnified by a telescope and imaged into a spectrometer for analysis. In addition to the technological barriers for remote detection, other challenges exist of using laser spectroscopy in the open field, such as potential laser-induced eye and skin damage [93]. Acute retinal damage can occur when the incident laser wavelength is greater than $400 \mathrm{~nm}[94,95]$, producing thermal damage to the retina as the lens focuses the intense laser beam into the eye through the virtuous humor. Retinal damage is reduced when laser wavelength is less than $400 \mathrm{~nm}$ because most of absorption occurs between the lens and the cornea. While prolonged exposure can cause cancer and further photochemical damage, instantaneous exposure is less harmful than damage caused with visible and IR lasers. Using a UV laser to perform proximal Raman spectroscopy further alleviates some of the challenges associated with signal detection at a distance. An added benefit of using UV lasers is that they operate in the "solar blind region" of wavelengths lower than $300 \mathrm{~nm}$. This alleviates much of the background radiation, which is blocked out by ozone. Finally, when probing with lasers below $250 \mathrm{~nm}$, fluorescence signals are outside of a typical Raman spectroscopy window of $4000 \mathrm{~cm}^{-1}$ [76].

Applications of proximal Raman for detecting low volatility agents such as explosives have their own set of challenges. The many sources for background signal in the ambient environment tend to inhibit Raman signal generation from low volatility explosive materials [96]. To overcome the inherently weak Raman signal, interference from ambient conditions, and the resulting unacceptable signal-to-noise ratio, proximal Raman is performed with pulsed lasers and gated intensified CCD detector. While most laboratory-based Raman lasers operate in continuous wave mode, it is disadvantageous to apply that same principle into the field, where the signal from the abundant background can overwhelm the collected data. A more effective way to collect spectra in a background-rich environment is to pulse the laser and gate the collection time of the CCD detector, thereby allowing only a fraction of the signal to reach the detector. Synching these pulses eliminates much of the background signal, allowing the system to collect data only during laser excitation. This "gated" approach reduced background contribution in pulsed geometries by a factor of 50,000 [76].

One can also incorporate Resonance Raman spectroscopy into a remote system to detect trace amounts of explosives at proximal distances. Currently, this type of work is being performed at Sweden's Defense Research Agency, or FOI, where they showed detection of various compounds in the standoff distance realm $(>200 \mathrm{~m})$. The researchers detected bulk explosives at $470 \mathrm{~m}$ in open space and $200 \mathrm{~m}$ through a window [97]. To combat diverging lasers at such large distances, they implemented a 1:5 laser beam expander. A telescope lens fed into a spectrometer using optical fibers was used to collect the data. The spectrometer was mounted with a gated intensified CCD to suppress the background signal from the ambient, similar to the technique used in [76]. Their resonance-enhanced Raman spectroscopy results showed that a $250 \mathrm{~nm}$ excitation laser source produced enhancement values of the Raman cross section ranging from 2,200 to 100,000 at standoff distance of $200-470 \mathrm{~m}$.

Another unexplored area of proximal Raman spectroscopy research is the implementation of SERS at a distance away from the sample. The use of SERS at remote distances 
is currently limited by the requirement that the potentially hazardous analyte of interest must be in close proximity to the SERS substrate. Several studies demonstrated limited ability for SERS in proximal mode. One group predeposited a SERS substrate with an analyte and then placed the prefabricated sample at a proximal distance for detection [83]. In another demonstration of portable SERS, a lowpower, battery-operated Inspector Raman spectrometer was used to identify Bacillus anthracis spores predeposited on a metal film-over-nanospheres (M-FON) SERS substrate [12]. Both demonstrations required artificial deposition of analyte over the SERS substrate. Applications of the SERS technique in the field would require the SERS substrate to collect the analyte from the environment at some distance from the Raman system and for the operator to monitor the loaded substrate remotely.

A useful deployment of the SERS method would be to capture analyte vapors through adsorption onto the SERS substrate. This is impractical in applications such as forensics, where low amounts of analytes are typically found. In application where a "cloud" of a CWA or a BWA is deployed, there may be enough analyte to produce a large signal. Vapor detection using SERS was recently demonstrated using vapor deposition onto engineered SERS substrates in gas chambers [98]. Additionally, chemical modification of SERS substrates resulted in enhanced oxidation protection for the substrate and improved chemoselectively of numerous volatile species [99].

4.3. SERS. Several research groups have attempted to enhance SERS' ability to detect analytes in environments that would simulate realistic conditions such as dispersing a dilute amount of sample in aqueous media. Innovative use of fiber optics and Raman signal enhancement reduced limits of detection for active salts [100] and proteins and bacteria in aqueous solutions [101]. Several firms have developed hybrid detectors incorporating SERS substrates in a novel fashion to enhance analyte sampling and improve system sensitivity. SpectraFluidics recently developed an open channel microfluidics detector for airborne vapors that uses SERS for analyte detection [102]. The SpectraFluidics system takes advantage of both the analyte concentration effects of the open microfluidic channel and the enhancement factor (EF) of analytes that become attached to the silver colloids used as the SERS substrate. The key enabling technology is termed free-surface fluidics (FSF), where one or more fluidic surfaces, confined by surface tension forces, are exposed to the surrounding atmosphere. Analyte is absorbed into the microchannel and concentrated by several orders of magnitude. The analyte interacts with colloidal nanoparticles, thereby producing a SERS spectrum. Microfluidics allows for deterministic control of SERS "hot spot" aggregation, which can increase normal Raman scattering signal strength by up to nine orders in magnitude. Operationally, the microchannel must be maintained at the dew point to prevent the eluent in the open microchannel from evaporating.

Real-Time Analyzers (RTAs) developed a SERS-based detector that can identify numerous drugs in saliva at $\mathrm{ng} / \mathrm{mL}$ concentrations within ten minutes. Eighty drugs of abuse and their metabolites were successfully measured by SERS using gold- and silver-doped sol-gels immobilized in glass capillaries [103]. In a focused study, a method was developed that provided consistent detection of $50 \mathrm{ppb}$ cocaine in saliva. This general method was successfully applied to the detection of a number of additional drugs in saliva, such as amphetamine, diazepam, and methadone. Identification was provided by matching measured spectra to a SERS library of over 150 different drugs, acquired specifically for the RTA portable SERS detector. Trace detection was accomplished using gold nanoparticles trapped within a porous glass matrix. The adsorbed analyte on the trapped gold nanoparticles generated the SERS enhancement since the molecules were in close proximity to the SERS hot spots. Speed is provided by a syringe driven sample system that extracts the drugs from saliva and provides SERS-activity.

4.4. Raman Chemical Imaging. Raman chemical imaging (RCI) combines microscopy techniques with Raman spectroscopy to form two-dimensional (2D) maps that spatially indicate the location of a chemical of interest. Uses of chemical imaging techniques include, but are not limited to, analyzing composite materials to determine concentration of a desired chemical species, creating two- or threedimensional "chemical maps" of samples, and analyzing the chemical composition of an unknown substance.

There are two general approaches to collecting Raman images: serial and direct imaging [104]. In the serial imaging technique, an area of interest is rastered with a laser beam in the $x$ and $y$ directions, similar to atomic force microscopy, by either moving the sample on a motorized stage or piezodriving the laser across a stationary sample [105]. In the serial mode, the entire Raman spectrum is obtained at each point. Another approach to serial imaging is line mapping, which occurs by rastering a horizontal laser line, whose length is the field of view, in the $x$ or $y$ directions. Again, the entire Raman spectrum is obtained at each line. In direct imaging, or wide field imaging, the entire field of view is illuminated by the incident laser. The Raman-scattered signal goes through a filter, such as a liquid crystal tunable filter (LCTF), which selects a desired frequency to input to a CCD detector. The image is recorded with many frequency increments and a hyperspectral cube is generated with two spatial axes and one spectral axis [106].

Chemical imaging has proven helpful in forensic fingerprint analysis. The ability to pinpoint the location of trace substances is an important asset in forensic analysis. Currently, there is great difficulty in identifying the location of an analyte in a collected fingerprint sample and determining its identity. It is very difficult to nondestructively determine whether a latent fingerprint contains residue of any illicit substance. Conventional Raman spectroscopy requires destruction of the fingerprint to create a powder containing the illicit residue $[18,19,107]$. The destruction would make it more difficult to match the fingerprint to the perpetrator identity for crime analysis and prosecution. Raman imaging solves this problem by allowing for visual and chemical identification of the chemical of interest at the same time $[106,108]$. The undisturbed fingerprint 
is examined under a microscope and suspected crystallites are immediately analyzed without disturbing the sample. Unfortunately, the chemical imaging technology is expensive and requires immobile equipment that currently does not satisfy in-field requirements. For example, it takes over 60 minutes to collect a chemical image of a typical fingerprint sample [106]. To date, the most common use of RCI has been limited to the laboratory.

A very recent study uniquely combined remote Raman spectroscopy and RCI to chemically image trace explosives [109]. Bulk explosive samples DNT, sulphur, TNT, and ammonium nitrate are placed next to each other at $10 \mathrm{~m}$ distance and imaged in direct mode. The Raman signal was collected in collimated form into a telescope, which then split the beam into two parts. One entered a standard CCD for collection of a white light image, while the other beam entered an LCFT. Discernible images were collected of individual explosive samples using reference spectra for each explosive type. While initially successful, it was determined that signal and performance could be further improved if UV lasers were used in subsequent studies [109].

Chemical imaging can also be performed using the coherent anti-Stokes Raman spectroscopy (CARS) approach. Because of the enhancement effects, the laser power required for CARS imaging is factor of 100 less than required by conventional Raman spectroscopy [110], eliminating much of the photodegregation of the sample and reducing fluorescence generation. In CARS microscopy applications, imaging takes place by the serial method. Shorter raster times are required to obtain an image when operating under resonance conditions. Both the pump and the probe laser beams are tightly focused on a point, yielding a small excitation volume $\left(<1 \mu \mathrm{m}^{3}\right)$, that is, good resolution [17]. The small volume allows for background rejection, less damage to sample, and three-dimensional microscopy by scanning different focal planes [110].

4.5. Heterodyne Imaging. Space exploration is also driving advancements in technology to make Raman spectroscopy more lightweight and robust, enhancing capabilities to detect and characterize organic and biological materials. Michael Angel reported a promising spectrometer design, which contains no moving parts, used primarily for planetary applications, the spatial heterodyne interferometer. The spatial heterodyne spectrometer (SHS) is compatible with pulsed laser excitation and offers several advantages, including high spectral resolution that is limited only by the diffraction gratings, in a very small form factor; very high optical etendue and thus high throughput; and demonstrated high resolution in the UV [111]. Spatial heterodyne spectrometers have been applied predominantly in astronomy and have not been reported previously for Raman applications, most likely because SHS technology has been focused on astronomical remote sensing and because most systems are designed for a very small spectral band pass. The Angel team made proof-of-principal Raman measurements on liquid and solid samples with an SHS Raman spectrometer using visible $(532 \mathrm{~nm})$ laser excitation; the overall sensitivity and light throughput of the system have not yet been determined.
Visible excitation was used in this proof of concept study, but the goal is to design a deep-UV SHS Raman system to gain the improved sensitivity of short wavelength excitation. The inherent robustness of the SHS system and its recent transition for astronomical to stand-off chemical detection applications suggest that this approach could substantially improve the performance of fielded Raman detection systems.

\section{Conclusion}

Raman spectroscopy is a useful technique for identifying potentially hazardous materials for homeland security and defense applications. The technique does not require intimate contact with a sample and does not require extensive sample preparation, therefore preserving sample material integrity. These are important factors if true identity of hazardous material is required or is needed for forensic analysis and, subsequently, as evidence. Additionally, the technique is able to identify hazards unambiguously, only requiring a robust spectral library to identify the analyte. Such spectrum libraries are constantly in development and in the state of constant updating. The basic Raman techniques reviewed here, which are useful for identifying hazardous materials, are conventional Raman, SERS, SORS, TORS, and resonance Raman. The functionality of these techniques can be enhanced as they are integrated with new emerging technologies, which can be used to exploit the Raman effect in microscopy, remote detection, and spectral imaging.

\section{References}

[1] M. J. Pelletier, Analytical Applications of Raman Spectroscopy, Blackwell Science, 1999.

[2] R. S. Das and Y. K. Agrawal, "Raman spectroscopy: recent advancements, techniques and applications," Vibrational Spectroscopy, vol. 57, no. 2, pp. 163-176, 2011.

[3] K. Y. Noonan, L. A. Tonge, O. S. Fenton, D. B. Damiano, and K. A. Frederick, "Rapid classification of simulated street drug mixtures using Raman spectroscopy and principal component analysis," Applied Spectroscopy, vol. 63, no. 7, pp. 742-747, 2009.

[4] S. D. Harvey, M. E. Vucelick, R. N. Lee, and B. W. Wright, "Blind field test evaluation of Raman spectroscopy as a forensic tool," Forensic Science International, vol. 125, no. 1, pp. 12-21, 2002.

[5] M. B. Denton, R. P. Sperline, J. H. Giles, D. A. Gilmore, C. J. S. Pommier, and R. T. Downs, "Advances in the application of array detectors for improved chemical analysis, part I. Comparison of qualitative analyses using large, computer-based Raman spectral libraries," Australian Journal of Chemistry, vol. 56, no. 2-3, pp. 117-131, 2003.

[6] R. S. Golightly, W. E. Doering, and M. J. Natan, "Surfaceenhanced Raman spectroscopy and homeland security: a perfect match?" ACS Nano, vol. 3, no. 10, pp. 2859-2869, 2009.

[7] M. L. Lewis, I. R. Lewis, and P. R. Griffiths, Infrared and Raman Spectroscopy in Forensic Science, John Wiley \& Sons, 2012. 
[8] K. Carron and R. Cox, "Qualitative analysis and the answer box: a perspective on portable Raman spectroscopy," Analytical Chemistry, vol. 82, no. 9, pp. 3419-3425, 2010.

[9] E. V. Efremov, F. Ariese, and C. Gooijer, "Achievements in resonance Raman spectroscopy. Review of a technique with a distinct analytical chemistry potential," Analytica Chimica Acta, vol. 606, no. 2, pp. 119-134, 2008.

[10] M. D. Hargreaves, K. Page, T. Munshi, R. Tomsett, G. Lynch, and H. G. M. Edwards, "Analysis of seized drugs using portable Raman spectroscopy in an airport environment-a proof of principle study," Journal of Raman Spectroscopy, vol. 39, no. 7, pp. 873-880, 2008.

[11] C. Eliasson and P. Matousek, "Noninvasive authentication of pharmaceutical products through packaging using spatially offset Raman spectroscopy," Analytical Chemistry, vol. 79, no. 4, pp. 1696-1701, 2007.

[12] X. Zhang, M. A. Young, O. Lyandres, and R. P. Van Duyne, "Rapid detection of an anthrax biomarker by surfaceenhanced Raman spectroscopy," Journal of the American Chemical Society, vol. 127, no. 12, pp. 4484-4489, 2005.

[13] M. Bloomfield, P. W. Loeffen, and P. Matousek, "Detection of concealed substances in sealed opaque plastic and coloured glass containers using SORS," in Optics and Photonics for Counterterrorism and Crime Fighting VI and Optical Materials in Defence Systems Technology VII, vol. 7838 of Proceedings of SPIE, September 2010.

[14] P. Matousek, I. P. Clark, E. R. C. Draper et al., "Subsurface probing in diffusely scattering media using spatially offset Raman spectroscopys," Applied Spectroscopy, vol. 59, no. 4, pp. 393-400, 2005.

[15] R. A. Halvorson and P. J. Vikesland, "Surface-enhanced Raman spectroscopy (SERS) for environmental analyses," Environmental Science and Technology, vol. 44, no. 20, pp. 7749-7755, 2010.

[16] C. L. Haynes, A. D. McFarland, and R. P. Van Duyne, "Surface-enhanced: Raman spectroscopy," Analytical Chemistry, vol. 77, no. 17, pp. 338A-346A, 2005.

[17] J. X. Cheng and X. S. Xie, "Coherent anti-Stokes Raman scattering microscopy: instrumentation, theory, and applications," Journal of Physical Chemistry B, vol. 108, no. 3, pp. 827-840, 2004.

[18] H. G. M. Edwards and J. S. Day, "Fourier transform Raman spectroscopic studies of the curing of cyanoacrylate glue," Journal of Raman Spectroscopy, vol. 35, no. 7, pp. 555-560, 2004.

[19] H. G. M. Edwards and J. S. Day, "Anomalies in polycyanoacrylate formation studied by Raman spectroscopy: implications for the forensic enhancement of latent fingerprints for spectral analysis," Vibrational Spectroscopy, vol. 41, no. 2, pp. 155-159, 2006.

[20] J. W. Chan, A. P. Esposito, C. E. Talley, C. W. Hollars, S. M. Lane, and T. Huser, "Reagentless identification of single bacterial spores in aqueous solution by confocal laser tweezers raman spectroscopy," Analytical Chemistry, vol. 76, no. 3, pp. 599-603, 2004.

[21] N. Jamin, P. Dumas, J. Moncuit et al., "Highly resolved chemical imaging of living cells by using synchrotron infrared microspectrometry," Proceedings of the National Academy of Sciences of the United States of America, vol. 95, no. 9, pp. 4837-4840, 1998.

[22] B. K. Dable, B. A. Love, T. M. Battaglia, K. S. Booksh, M. D. Lilley, and B. J. Marquardt, "Characterization and quantitation of a tertiary mixture of salts by Raman spectroscopy in simulated hydrothermal vent fluid," Applied Spectroscopy, vol. 60, no. 7, pp. 773-780, 2006.

[23] M. N. Leger and A. G. Ryder, "Comparison of derivative preprocessing and automated polynomial baseline correction method for classification and quantification of narcotics in solid mixtures," Applied Spectroscopy, vol. 60, no. 2, pp. 182193, 2006.

[24] T. G. Matthews, "Blank limitations in laser excited solution luminescence," Analytical Chemistry, vol. 51, no. 4, pp. 583$585,1979$.

[25] B. Schrader, B. Dippel, S. Fendel et al., "NIR FT Raman spectroscopy-a new tool in medical diagnostics," Journal of Molecular Structure, vol. 408-409, pp. 23-31, 1997.

[26] B. Schrader, B. Dippel, I. Erb et al., "NIR Raman spectroscopy in medicine and biology: results and aspects," Journal of Molecular Structure, vol. 480-481, pp. 21-32, 1999.

[27] M. G. Albrecht and J. A. Creighton, "Anomalously intense Raman spectra of pyridine at a silver electrode," Journal of the American Chemical Society, vol. 99, no. 15, pp. 5215-5217, 1977.

[28] M. Fleischmann, P. J. Hendra, and A. J. McQuillan, "Raman spectra of pyridine adsorbed at a silver electrode," Chemical Physics Letters, vol. 26, no. 2, pp. 163-166, 1974.

[29] J. A. Dieringer, A. D. McFarland, N. C. Shah et al., "Surface enhanced Raman spectroscopy: new materials, concepts, characterization tools, and applications," Faraday Discussions, vol. 132, pp. 9-26, 2006.

[30] H. Xu, J. Aizpurua, M. Käll, and P. Apell, "Electromagnetic contributions to single-molecule sensitivity in surfaceenhanced Raman scattering," Physical Review E, vol. 62, no. 3 B, pp. 4318-4324, 2000.

[31] B. Pietrobon, M. McEachran, and V. Kitaev, "Synthesis of size-controlled faceted pentagonal silver nanorods with tunable plasmonic properties and self-assembly of these nanorods," ACS Nano, vol. 3, no. 1, pp. 21-26, 2009.

[32] J. P. Kottmann, O. J. F. Martin, D. R. Smith, and S. Schultz, "Dramatic localized electromagnetic enhancement in plasmon resonant nanowires," Chemical Physics Letters, vol. 341, no. 1-2, pp. 1-6, 2001.

[33] M. Micic, N. Klymyshyn, Y. D. Suh, and H. P. Lu, "Finite element method simulation of the field distribution for AFM tip-enhanced surface-enhanced Raman scanning microscopy," Journal of Physical Chemistry B, vol. 107, no. 7, pp. 1574-1584, 2003.

[34] S. H. Christiansen, M. Becker, S. Fahlbusch et al., "Signal enhancement in nano-Raman spectroscopy by gold caps on silicon nanowires obtained by vapour-liquid-solid growth," Nanotechnology, vol. 18, no. 3, Article ID 035503, 2007.

[35] N. A. Abu Hatab, J. M. Oran, and M. J. Sepaniak, "Surfaceenhanced Raman spectroscopy substrates created via electron beam lithography and nanotransfer printing," ACS Nano, vol. 2, no. 2, pp. 377-385, 2008.

[36] E. Altewischer, M. P. Van Exter, and J. P. Woerdman, "Plasmon-assisted transmission of entangled photons," Nature, vol. 418, no. 6895, pp. 304-306, 2002.

[37] T. W. Ebbesen, H. J. Lezec, H. F. Ghaemi, T. Thio, and P. A. Wolff, "Extraordinary optical transmission through subwavelenght hole arrays," Nature, vol. 391, no. 6668, pp. 667669, 1998.

[38] A. A. Tseng, "Recent developments in nanofabrication using focused ion beams," Small, vol. 1, no. 10, pp. 924-939, 2005.

[39] R. Que, M. Shao, S. Zhuo, C. Wen, S. Wang, and S.-T. Lee, "Highly reproducible surface-enhanced raman scattering on 
a capillarity-assisted gold nanoparticle assembly," Advanced Functional Materials, vol. 21, no. 17, pp. 3337-3343, 2011.

[40] Y. Wang, M. Becker, L. Wang et al., "Nanostructured gold films for SERS by block copolymer-templated galvanic displacement reactions," Nano Letters, vol. 9, no. 6, pp. 2384 2389, 2009.

[41] A. Sánchez-Iglesias, P. Aldeanueva-Potel, W. Ni et al., "Chemical seeded growth of Ag nanoparticle arrays and their application as reproducible SERS substrates," Nano Today, vol. 5, no. 1, pp. 21-27, 2010.

[42] W. Lee, S. Y. Lee, R. M. Briber, and O. Rabin, "Self-assembled SERS substrates with tunable surface plasmon resonances," Advanced Functional Materials, vol. 21, no. 18, pp. 34243429, 2011.

[43] A. Gopinath, S. V. Boriskina, B. M. Reinhard, and L. Dal Negro, "Deterministic aperiodic arrays of metal nanoparticles for surface-enhanced Raman scattering (SERS)," Optics Express, vol. 17, no. 5, pp. 3741-3753, 2009.

[44] W. J. Cho, Y. Kim, and J. K. Kim, "Ultrahigh-density array of silver nanoclusters for SERS substrate with high sensitivity and excellent reproducibility," ACS Nano, vol. 6, no. 1, pp. 249-255, 2012.

[45] B. Yan, A. Thubagere, W. R. Premasiri, L. D. Ziegler, L. D. Negro, and B. M. Reinhard, "Engineered SERS substrates with multiscale signal enhancement: nanoparticle cluster arrays," ACS Nano, vol. 3, no. 5, pp. 1190-1202, 2009.

[46] L. Yang, B. Yan, W. R. Premasiri, L. D. Ziegler, L. D. Negro, and B. M. Reinhard, "Engineering nanoparticle cluster arrays for bacterial biosensing: the role of the building block in multiscale SERS substrates," Advanced Functional Materials, vol. 20, no. 16, pp. 2619-2628, 2010.

[47] Z. Q. Tian, B. Ren, and D. Y. Wu, "Surface-enhanced Raman scattering: from noble to transition metals and from rough surfaces to ordered nanostructures," Journal of Physical Chemistry B, vol. 106, no. 37, pp. 9463-9483, 2002.

[48] H. Ko, S. Chang, and V. V. Tsukruk, "Porous substrates for label-free molecular level detection of nonresonant organic molecules," ACS Nano, vol. 3, no. 1, pp. 181-188, 2009.

[49] H. Yan, C. Gu, C. Yang et al., "Hollow core photonic crystal fiber surface-enhanced Raman probe," Applied Physics Letters, vol. 89, no. 20, Article ID 204101, 2006.

[50] T. A. Alexander, "Development of methodology based on commercialized SERS-active substrates for rapid discrimination of Poxviridae virions," Analytical Chemistry, vol. 80, no. 8, pp. 2817-2825, 2008.

[51] T. A. Alexander and D. M. Le, "Characterization of a commercialized SERS-active substrate and its application to the identification of intact Bacillus endospores," Applied Optics, vol. 46, no. 18, pp. 3878-3890, 2007.

[52] T. A. Alexander, "Applications of Surface-Enhanced Raman Spectroscopy (SERS) for biosensing: an analysis of reproducible, commercially available substrates," in Smart Medical and Biomedical Sensor Technology III, vol. 6007 of Proceedings of SPIE, Boston, Mass, USA, October 2005.

[53] T. A. Alexander, P. M. Pellegrino, and J. B. Gillespie, "Near-infrared surface-enhanced-Raman-scatteringmediated detection of single optically trapped bacterial spores," Applied Spectroscopy, vol. 57, no. 11, pp. 1340-1345, 2003.

[54] T. A. Alexander and A. E. Wickenden, "Development of metallic nanostructures for biosensing," in Chemical and Biological Sensing VI, vol. 5795 of Proceedings of SPIE, pp. 3038, Orlando, Fla, USA, March 2005.
[55] M. E. Hankus, D. N. Stratis-Cullum, and P. M. Pellegrino, "Enabling Technologies for Point and Remote Sensing of Chemical and Biological Agents Using Surface Enhanced Raman Scattering (SERS) Techniques," Tech. Rep., U.S. Army Research Laboratory, 2009.

[56] M. E. Hankus, D. N. Stratis-Cullum, and P. M. Pellegrino, "Characterization of next-generation commercial surfaceenhanced Raman scattering (SERS) substrates," in Chemical, Biological, Radiological, Nuclear, and Explosives (CBRNE) Sensing XII, vol. 8018 of Proceedings of SPIE, April 2011.

[57] G. Comanescu, C. K. Manka, J. Grun, S. Nikitin, and D. Zabetakis, "Identification of explosives with twodimensional ultraviolet resonance Raman spectroscopy," Applied Spectroscopy, vol. 62, no. 8, pp. 833-839, 2008.

[58] P. Jander and R. Noll, "Automated detection of fingerprint traces of high explosives using ultraviolet raman spectroscopy," Applied Spectroscopy, vol. 63, no. 5, pp. 559-563, 2009.

[59] L. Nagli, M. Gaft, Y. Fleger, and M. Rosenbluh, "Absolute Raman cross-sections of some explosives: trend to UV," Optical Materials, vol. 30, no. 11, pp. 1747-1754, 2008.

[60] D. D. Tuschel, A. V. Mikhonin, B. E. Lemoff, and S. A. Asher, "Deep ultraviolet resonance raman excitation enables explosives detection," Applied Spectroscopy, vol. 64, no. 4, pp. 425-432, 2010.

[61] D. R. Felt, S. L. Larson, and E. J. Valente, "UV-VIS spectroscopy of 2,4,6-trinitrotoluene-hydroxide reaction," Chemosphere, vol. 49, no. 3, pp. 287-295, 2002.

[62] S. A. Oladepo, K. Xiong, Z. Hong, and S. A. Asher, "Elucidating peptide and protein structure and dynamics: UV resonance raman spectroscopy," Journal of Physical Chemistry Letters, vol. 2, no. 4, pp. 334-344, 2011.

[63] Z. Chi, X. G. Chen, J. S. W. Holtz, and S. A. Asher, "Uv resonance raman-selective amide vibrational enhancement: quantitative methodology for determining protein secondary structure," Biochemistry, vol. 37, no. 9, pp. 2854-2864, 1998.

[64] Y. Y. Wang, F. Couny, P. S. Light, B. J. Mangan, and F. Benabid, "Compact and portable multiline UV and visible Raman lasers in hydrogen-filled HC-PCF," Optics Letters, vol. 35, no. 8, pp. 1127-1129, 2010.

[65] R. F. Begley, A. B. Harvey, and R. L. Byer, "Coherent antiStokes Raman spectroscopy," Applied Physics Letters, vol. 25, no. 7, pp. 387-390, 1974.

[66] D. Pestov, R. K. Murawski, G. O. Ariunbold et al., "Optimizing the laser-pulse configuration for coherent Raman spectroscopy," Science, vol. 316, no. 5822, pp. 265-268, 2007.

[67] W. M. Tolles, J. W. Nibler, J. R. McDonald, and A. B. Harvey, "A review of the theory and application of Coherent AntiStokes Raman Spectroscopy (CARS)," Applied Spectroscopy, vol. 31, no. 4, pp. 253-272, 1977.

[68] A. M. Zheltikov, "Coherent anti-stokes Raman scattering: from proof-of-the-principle experiments to femtosecond CARS and higher order wave-mixing generalizations," Journal of Raman Spectroscopy, vol. 31, no. 8-9, pp. 653-667, 2000.

[69] O. Katz, A. Natan, Y. Silberberg, and S. Rosenwaks, "Standoff detection of trace amounts of solids by nonlinear Raman spectroscopy using shaped femtosecond pulses," Applied Physics Letters, vol. 92, no. 17, Article ID 171116, 2008.

[70] M. T. Bremer, P. J. Wrzesinski, N. Butcher, V. V. Lozovoy, and M. Dantus, "Highly selective standoff detection and imaging of trace chemicals in a complex background using 
single-beam coherent anti-Stokes Raman scattering," Applied Physics Letters, vol. 99, no. 10, Article ID 101109, 2011.

[71] D. Oron, N. Dudovich, and Y. Silberberg, "Single-pulse phase-contrast nonlinear Raman spectroscopy," Physical Review Letters, vol. 89, no. 27, pp. 2730011-2730014, 2002.

[72] H. Li, D. A. Harris, B. Xu, P. J. Wrzesinski, V. V. Lozovoy, and M. Dantus, "Standoff and arms-length detection of chemicals with single-beam coherent anti-Stokes Raman scattering," Applied Optics, vol. 48, no. 4, pp. B17-B22, 2009.

[73] A. Portnov, I. Bar, and S. Rosenwaks, "Highly sensitive standoff detection of explosives via backward coherent antiStokes Raman scattering," Applied Physics B, vol. 98, no. 2-3, pp. 529-535, 2010.

[74] P. Matousek and A. W. Parker, "Non-invasive probing of pharmaceutical capsules using transmission Raman spectroscopy," Journal of Raman Spectroscopy, vol. 38, no. 5, pp. 563-567, 2007.

[75] P. Matousek, "Deep non-invasive Raman spectroscopy of living tissue and powders," Chemical Society Reviews, vol. 36, no. 8, pp. 1292-1304, 2007.

[76] A. K. Misra, S. K. Sharma, C. H. Chio, P. G. Lucey, and B. Lienert, "Pulsed remote Raman system for daytime measurements of mineral spectra," Spectrochimica Acta A, vol. 61, no. 10, pp. 2281-2287, 2005.

[77] P. Matousek, N. Everall, M. Towrie, and A. W. Parker, “Depth profiling in diffusely scattering media using Raman spectroscopy and picosecond Kerr gating," Applied Spectroscopy, vol. 59, no. 2, pp. 200-205, 2005.

[78] M. D. Hargreaves, N. A. Macleod, V. L. Brewster, T. Munshi, H. G. M. Edwards, and P. Matousek, "Application of portable Raman spectroscopy and benchtop spatially offset Raman spectroscopy to interrogate concealed biomaterials," Journal of Raman Spectroscopy, vol. 40, no. 12, pp. 1875-1880, 2009.

[79] P. W. Loeffen, G. Maskall, S. Bonthron, M. Bloomfield, C. Tombling, and P. Matousek, "Chemical and explosives point detection through opaque containers using Spatially Offset Raman Spectroscopy (SORS)," in Chemical, Biological, Radiological, Nuclear, and Explosives (CBRNE) Sensing XII, vol. 8018 of Proceedings of SPIE, Orlando, Fla, USA, April 2011.

[80] P. W. Loeffen, G. Maskall, S. Bonthron, M. Bloomfield, C. Tombling, and P. Matousek, "Spatially offset Raman spectroscopy (SORS) for liquid screening," in Optics and Photonics for Counterterrorism and Crime Fighting VII; Optical Materials in Defence Systems Technology VIII; and QuantumPhysics-based Information Security, vol. 8189 of Proceedings of the SPIE, Prague, Czech Republic, September 2011.

[81] W. J. Olds, E. Jaatinen, P. Fredericks, B. Cletus, H. Panayiotou, and E. L. Izake, "Spatially offset Raman spectroscopy (SORS) for the analysis and detection of packaged pharmaceuticals and concealed drugs," Forensic Science International, vol. 212, pp. 69-77, 2011.

[82] B. L. Volodin, S. V. Dolgy, E. D. Melnik, E. Downs, J. Shaw, and V. S. Ban, "Wavelength stabilization and spectrum narrowing of high-power multimode laser diodes and arrays by use of volume Bragg gratings," Optics Letters, vol. 29, no. 16, pp. 1891-1893, 2004.

[83] J. P. Scaffidi, M. K. Gregas, B. Lauly, J. Chance Carter, S. Michael Angel, and T. Vo-Dinh, "Trace molecular detection via surface-enhanced raman scattering and surface-enhanced resonance raman scattering at a distance of 15 meters," Applied Spectroscopy, vol. 64, no. 5, pp. 485-492, 2010.
[84] L. F. Capitán-Vallvey and A. J. Palma, "Recent developments in handheld and portable optosensing-A review," Analytica Chimica Acta, vol. 696, no. 1-2, pp. 27-46, 2011.

[85] D. S. Moore and R. J. Scharff, "Portable Raman explosives detection," Analytical and Bioanalytical Chemistry, vol. 393, no. 6-7, pp. 1571-1578, 2009.

[86] H. Markert, J. Ring, N. Campbell, and K. Grates, A Comparison of Four Commercially Available Portable Raman Spectrometers, National Forensic Science Technology Center, 2011.

[87] S. Wallin, A. Pettersson, H. Östmark, and A. Hobro, "Laserbased standoff detection of explosives: a critical review," Analytical and Bioanalytical Chemistry, vol. 395, no. 2, pp. 259-274, 2009.

[88] J. C. Carter, S. M. Angel, M. Lawrence-Snyder, J. Scaffidi, R. E. Whipple, and J. G. Reynolds, "Standoff detection of high explosive materials at 50 meters in ambient light conditions using a small Raman instrument," Applied Spectroscopy, vol. 59, no. 6, pp. 769-775, 2005.

[89] W. M. P. Petryk, Proximal Detection, Defence Research and Development Canada, 2010.

[90] J. A. Guicheteau, S. D. Christesen, A. Tripathi et al., "Proximal and point detection of contaminated surfaces using Raman spectroscopy," in Optics and Photonics for Counterterrorism and Crime Fighting VII; Optical Materials in Defence Systems Technology VIII; and Quantum-Physics-based Information Security, vol. 8189 of Proceedings of the SPIE, October 2011.

[91] L. C. Pacheco-Londoño, W. Ortiz-Rivera, O. M. PrimeraPedrozo, and S. P. Hernández-Rivera, "Vibrational spectroscopy standoff detection of explosives," Analytical and Bioanalytical Chemistry, vol. 395, no. 2, pp. 323-335, 2009.

[92] C. W. Van Neste, L. R. Senesac, and T. Thundat, "Standoff spectroscopy of surface adsorbed chemicals," Analytical Chemistry, vol. 81, no. 5, pp. 1952-1956, 2009.

[93] L. A. Skvortsov and E. M. Maksimov, "Application of laser photothermal spectroscopy for standoff detection of trace explosive residues on surfaces," Quantum Electronics, vol. 40, no. 7, pp. 565-578, 2010.

[94] W. L. Eberhard, "Eye-safe tracking of oil fog plumes by UV lidar," Applied Optics, vol. 22, no. 15, pp. 2282-2285, 1983.

[95] A. Lavrov, A. B. Utkin, R. Vilar, and A. Fernandes, "Application of lidar in ultraviolet, visible and infrared ranges for early forest fire detection," Applied Physics B, vol. 76, no. 1, pp. 87-95, 2003.

[96] R. B. Cundall, T. F. Palmer, and C. E. C. Wood, "Vapour pressure measurements on some organic high explosives," Journal of the Chemical Society, Faraday Transactions 1, vol. 74, pp. 1339-1345, 1978.

[97] A. Pettersson, S. Wallin, H. Östmark et al., "Explosives standoff detection using Raman spectroscopy: from bulk towards trace detection," in Detection and Sensing of Mines, Explosive Objects, and Obscured Targets XV, vol. 7664 of Proceedings of SPIE, Orlando, Fla, USA, April 2010.

[98] J. Wang, L. Yang, S. Boriskina, B. Yan, and B. M. Reinhard, "Spectroscopic ultra-trace detection of nitroaromatic gas vapor on rationally designed two-dimensional nanoparticle cluster arrays," Analytical Chemistry, vol. 83, no. 6, pp. 22432249, 2011.

[99] P. A. Mosier-Boss and S. H. Lieberman, "Detection of volatile organic compounds using surface enhanced Raman spectroscopy substrates mounted on a thermoelectric cooler," Analytica Chimica Acta, vol. 488, no. 1, pp. 15-23, 2003. 
[100] J. Yin, T. Wu, J. Song et al., "SERS-active nanoparticles for sensitive and selective detection of cadmium ion $\left(\mathrm{Cd}^{2+}\right)$," Chemistry of Materials, vol. 23, no. 21, pp. 4756-4764, 2011.

[101] X. Yang, C. Gu, F. Qian, Y. Li, and J. Z. Zhang, "Highly sensitive detection of proteins and bacteria in aqueous solution using surface-enhanced raman scattering and optical fibers," Analytical Chemistry, vol. 83, no. 15, pp. 5888-5894, 2011.

[102] S. Pennathur, C. D. Meinhart, and H. T. Soh, "How to exploit the features of microfluidics technology," Lab on a Chip, vol. 8, no. 1, pp. 20-22, 2008.

[103] F. Inscore, C. Shende, A. Sengupta, H. Huang, and S. Farquharson, "Detection of drugs of abuse in saliva by surfaceenhanced raman spectroscopy (SERS)," Applied Spectroscopy, vol. 65, no. 9, pp. 1004-1008, 2011.

[104] S. Schlücker, M. D. Schaeberle, S. W. Huffman, and I. W. Levin, "Raman microspectroscopy: a comparison of point, line, and wide-field imaging methodologies," Analytical Chemistry, vol. 75, no. 16, pp. 4312-4318, 2003.

[105] A. Volkmer, J. X. Cheng, and X. S. Xie, "Vibrational imaging with high sensitivity via epidetected coherent anti-stokes raman scattering microscopy," Physical Review Letters, vol. 87, no. 2, Article ID 023901, 2001.

[106] A. Tripathi, E. D. Emmons, P. G. Wilcox et al., "SemiAutomated detection of trace explosives in fingerprints on strongly interfering surfaces with raman chemical imaging," Applied Spectroscopy, vol. 65, no. 6, pp. 611-619, 2011.

[107] M. J. West and M. J. Went, "The spectroscopic detection of drugs of abuse in fingerprints after development with powders and recovery with adhesive lifters," Spectrochimica Acta A, vol. 71, no. 5, pp. 1984-1988, 2009.

[108] E. D. Emmons, A. Tripathi, J. A. Guicheteau, S. D. Christesen, and A. W. Fountain, "Raman chemical imaging of explosivecontaminated fingerprints," Applied spectroscopy, vol. 63, no. 11, pp. 1197-1203, 2009.

[109] H. Östmark, M. Nordberg, and T. E. Carlsson, "Stand-off detection of explosives particles by multispectral imaging Raman spectroscopy," Applied Optics, vol. 50, no. 28, pp. 5592-5599, 2011.

[110] A. Zumbusch, G. R. Holtom, and X. S. Xie, "Threedimensional vibrational imaging by coherent anti-Stokes Raman scattering," Physical Review Letters, vol. 82, no. 20, pp. 4142-4145, 1999.

[111] N. R. Gomer, C. M. Gordon, P. Lucey, S. K. Sharma, J. C. Carter, and S. M. Angel, "Raman spectroscopy using a spatial heterodyne spectrometer: proof of concept," Applied Spectroscopy, vol. 65, no. 8, pp. 849-857, 2011. 


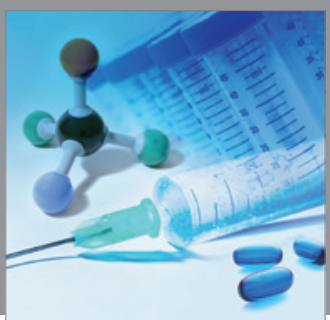

International Journal of

Medicinal Chemistry

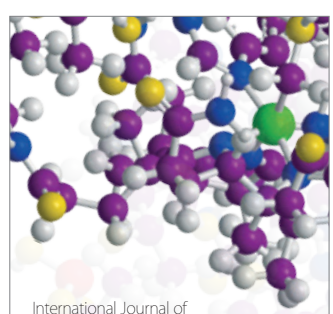

Carbohydrate Chemistry

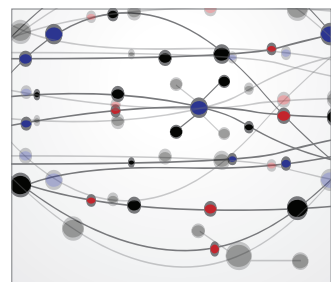

The Scientific World Journal
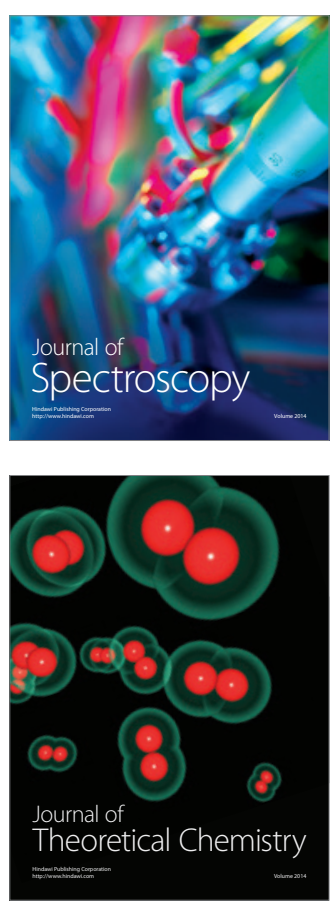
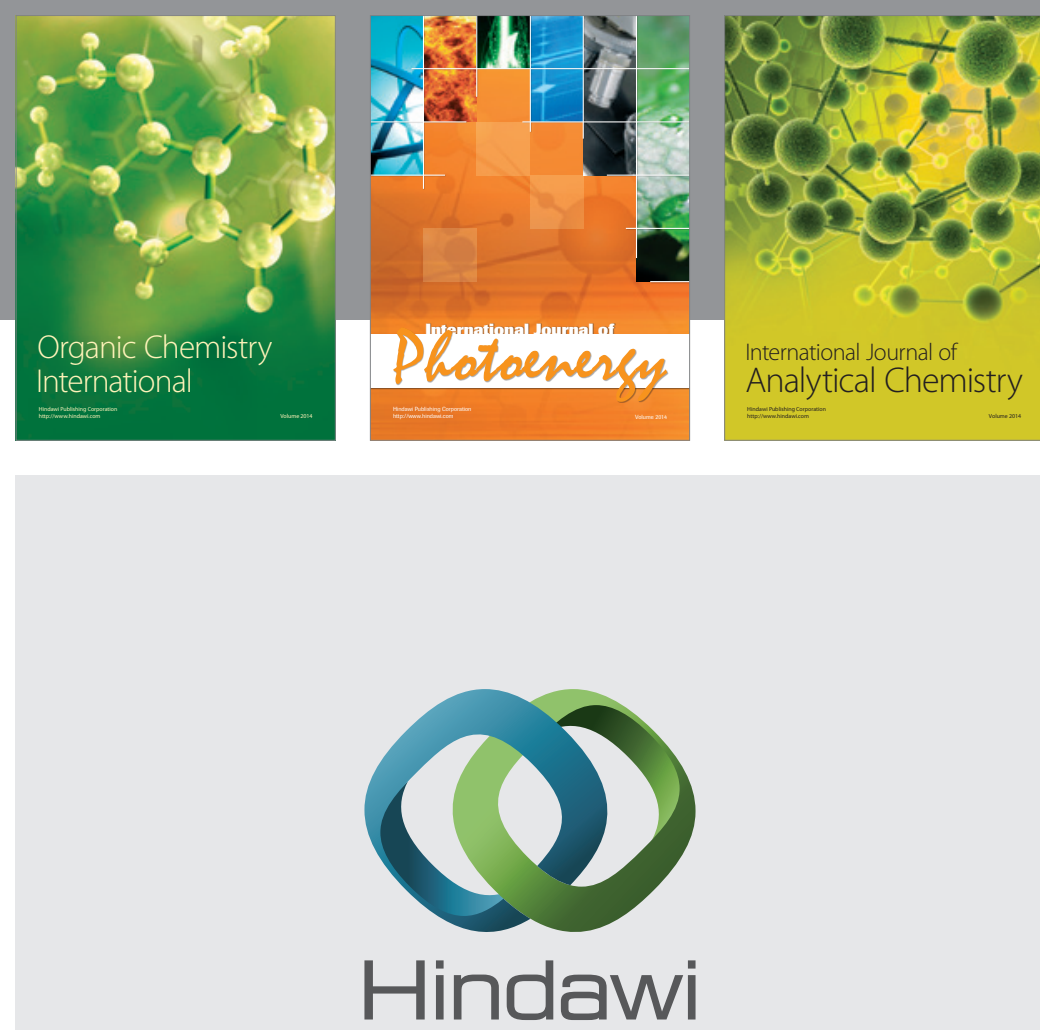

Submit your manuscripts at

http://www.hindawi.com
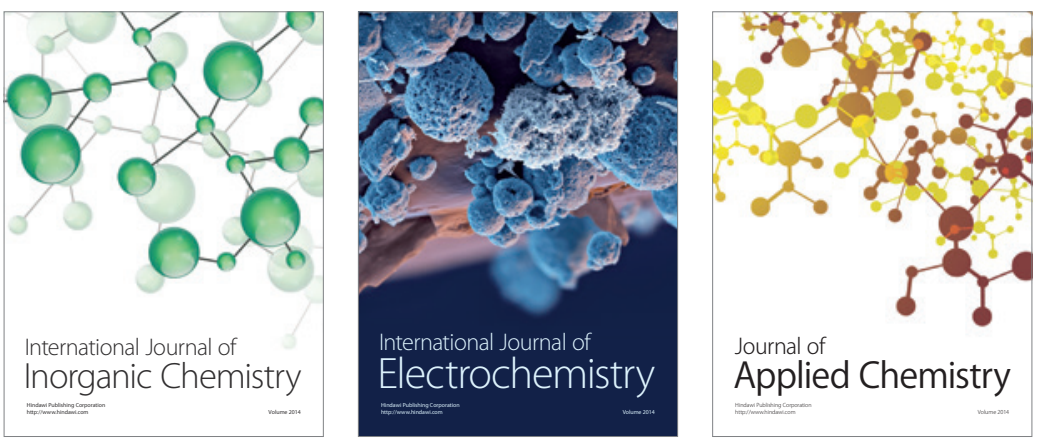

Journal of

Applied Chemistry
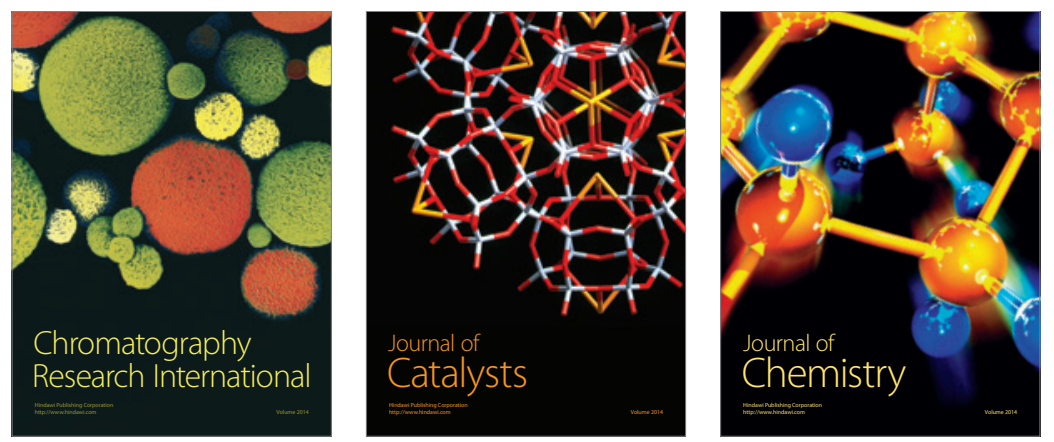
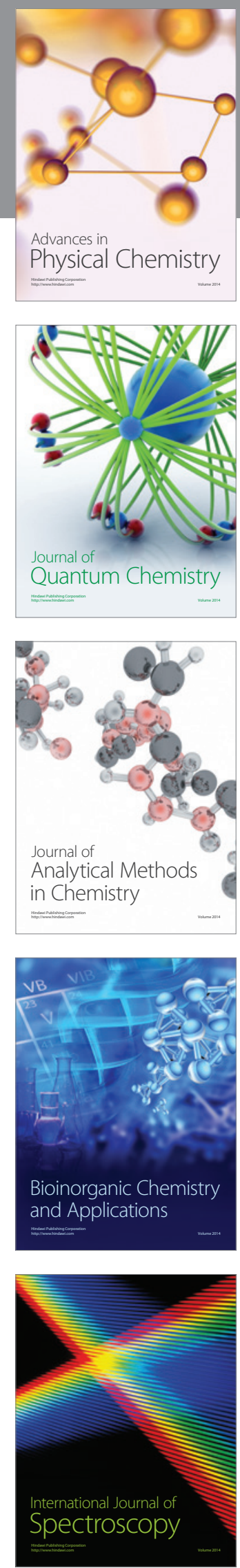